\title{
ON INVARIANT LINEAR MANIFOLDS ${ }^{1}$
}

\author{
P. A. FILLMORE ${ }^{2}$
}

\begin{abstract}
For a linear transformation $\boldsymbol{A}$ on a Banach space, let $\mathscr{L}(A)$ be the lattice of (not necessarily closed) invariant subspaces of $A$. For $A$ bounded it is shown that if $\mathscr{L}(A \oplus A) \subset \mathscr{L}(T \oplus T)$, or if $\mathscr{L}(A) \subset \mathscr{L}(T)$ and $T$ commutes with $A$, then $T$ is a polynomial in $A$. In the case of a Hilbert space, if $\mathscr{L}(A) \subset \mathscr{L}\left(A^{*}\right)$ then $A^{*}$ is a polynomial in $A$.
\end{abstract}

Introduction. A linear transformation $T$ on a vector space $V$ is algebraic if there is a nonzero polynomial $p$ such that $p(T)=0$; it is locally algebraic if for each $x \in V$ there is a nonzero polynomial $p$ (depending on $x$ ) such that $p(T) x=0$. A locally algebraic transformation need not be algebraic, but Kaplansky has shown [4, Theorem 15] that a bounded locally algebraic transformation on a Banach space must be algebraic. In this note we consider extensions of this fact and some related matters.

1. Specifically, let $A$ and $T$ be linear transformations on $V$ such that $T$ is locally a polynomial in $A$; that is, for each $x \in V$ there is a polynomial $p$ (depending on $x$ ) with $T x=p(A) x$. Must $T$ then be a polynomial in $A$ ? This question may be reformulated as follows: For any linear transformation $S$ on $V$, let

$$
\mathscr{L}(S)=\{M \mid M \text { is a subspace with } S M \subset M\},
$$

the lattice of invariant subspaces of $S$. Now observe that $T$ is locally a polynomial in $A$ if and only if $\mathscr{L}(A) \subset \mathscr{L}(T)$ (for if $A M \subset M$, then $p(A) M \subset$ $M$ for all polynomials $p$, so that when $T$ is locally a polynomial in $A$ we have $T M \subset M$; on the other hand, if $x \in V$ and

$$
M_{x}=\{p(A) x \mid p \text { is a polynomial }\},
$$

then $x \in M_{x} \in \mathscr{L}(A)$, so that when $\mathscr{L}(A) \subset \mathscr{L}(T)$ we get $x \in M_{x} \in \mathscr{L}(T)$, $T x \in M_{x}$, and $T x=p(A) x$ for some polynomial $\left.p\right)$. Thus our question becomes: does $\mathscr{L}(A) \subset \mathscr{L}(T)$ imply that $T$ is a polynomial in $A$ ? The

Received by the editors February 12, 1971.

AMS (MOS) subject classifications (1970). Primary 47A15, 47A65.

Key words and phrases. Invariant subspace lattice, locally algebraic operator.

${ }^{1}$ Research partially supported by a grant from the National Science Foundation.

2 This work was done while the author was a visiting professor at the University of Toronto.

(c) American Mathematical Society 1973 
answer is no, even for bounded transformations on a Banach space. For a simple example, take

$$
A=\left(\begin{array}{ll}
0 & 1 \\
0 & 0
\end{array}\right) \text { and } T=\left(\begin{array}{ll}
1 & 0 \\
0 & 2
\end{array}\right)
$$

on a two-dimensional space.

Motivated by the fact that a locally algebraic transformation is algebraic on each finite-dimensional subspace, we next ask whether $T$ must be a polynomial in $A$ whenever it is a polynomial in $A$ on each finitedimensional subspace. Again this is false, but we will show that the analogue of Kaplansky's Theorem holds. Actually a little more is true:

THEOREM 1. Let $A$ and $T$ be linear transformations on a Banach space $V$, with $A$ bounded. If $T$ is a polynomial in $A$ on each two-dimensional subspace of $V$, then $T$ is a polynomial in $A$.

Before proving this, a few remarks are in order. For any linear transformation $S$ on $V$, let $S^{(2)}=S \oplus S$ acting on $V \oplus V$. Then, as above, $T$ is a polynomial in $A$ on each two-dimensional subspace if and only if $\mathscr{L}\left(A^{(2)}\right) \subset \mathscr{L}\left(T^{(2)}\right)$. For a family $\mathscr{S}$ of linear transformations, let

$$
\begin{aligned}
\mathscr{S}^{(2)} & =\left\{S^{(2)} \mid S \in \mathscr{S}\right\}, \quad \text { and } \\
\mathscr{L}(\mathscr{S}) & =\{M \mid M \in \mathscr{L}(S) \text { for all } S \in \mathscr{S}\} .
\end{aligned}
$$

A general question of the type contemplated in the theorem goes as follows: For which algebras $\mathscr{A}$ of linear transformations is it true that $\mathscr{L}\left(\mathscr{A}^{(2)}\right) \subset \mathscr{L}\left(T^{(2)}\right)$ implies $T \in \mathscr{A}$ ? (For Theorem 1 take $\mathscr{A}$ to be all polynomials in $A$.)

LemMa. If $\mathscr{A}$ is an algebra with a separating vector, then $\mathscr{L}\left(\mathscr{A}^{(2)}\right) \subset$ $\mathscr{L}\left(T^{(2)}\right)$ implies $T \in \mathscr{A}$.

Proof. Let $x_{0}$ be a separating vector, so that $A \in \mathscr{A}$ and $A x_{0}=0$ imply $A=0$. Fix $A_{0} \in \mathscr{A}$ with $T x_{0}=A_{0} x_{0}$. If $y$ is any vector, by hypothesis there exists $A \in \mathscr{A}$ such that $T x_{0}=A x_{0}$ and $T y=A y$. Then $\left(A-A_{0}\right) x_{0}=0$ so $A=A_{0}$ and $T y=A_{0} y$. Since $y$ is arbitrary, $T=A_{0}$, as required.

Proof of TheORem. We need only show that for any bounded linear transformation $A$ on a Banach space, the algebra of polynomials in $A$ has a separating vector. Suppose first that $A$ is locally algebraic, so that $A$ is algebraic by Kaplansky's theorem. If $m$ is the minimum polynomial of $A$, it is easy to see (cf. Kaplansky's proof) that there is a vector $x_{0}$ at which the local minimum polynomial is $m$. Thus if $p(A) x_{0}=0$ then $m$ divides $p$, and so $p(A)=0$. Hence $x_{0}$ is the required separating vector. If $A$ is not locally algebraic, there is a vector $x_{0}$ such that $p(A) x_{0}=0$ implies $p=0$, and again we have a separating vector. 
Remarks. (i) As the proof shows, the theorem remains true without the topological hypotheses except when $A$ is locally algebraic but not algebraic. To see that this case is an exception, let $V=V_{2} \oplus V_{3} \oplus \cdots$ (algebraic direct sum) with $V_{n}$ an $n$-dimensional vector space, let $J_{n}$ be the $n \times n$ Jordan cell

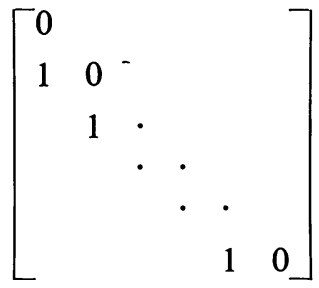

acting in $V_{n}$, and let $A=J_{2} \oplus J_{3} \oplus \cdots$. Then

$$
T=J_{2} \oplus\left(J_{3}+J_{3}^{2}\right) \oplus\left(J_{4}+J_{4}^{2}+J_{4}^{3}\right) \oplus \cdots
$$

satisfies $\mathscr{L}\left(A^{(n)}\right) \subset \mathscr{L}\left(T^{(n)}\right)$ for all $n$, and yet $T$ is not a polynomial in $A$.

(ii) Recall the question raised before the lemma: In what circumstances does $\mathscr{L}\left(\mathscr{A}^{(2)}\right) \in \mathscr{L}\left(T^{(2)}\right)$ imply $T \in \mathscr{A}$ ? For $n \geqq 1$ the $n$-closure of $\mathscr{A}$ is defined by

$$
C_{n}(\mathscr{A})=\left\{T \mid \mathscr{L}\left(\mathscr{A}^{(n)}\right) \subset \mathscr{L}\left(T^{(n)}\right)\right\}
$$

and the strict closure by

$$
C_{\infty}(\mathscr{A})=\bigcap_{n=1}^{\infty} C_{n}(\mathscr{A}) \text {. }
$$

Obviously $C_{1}(\mathscr{A}) \supset C_{2}(\mathscr{A}) \supset \cdots \supset C_{\infty}(\mathscr{A}) \supset \mathscr{A}$. If $\mathscr{A}^{\prime}$ is the commutant of $\mathscr{A}$ (the algebra of linear transformations that commute with every member of $\mathscr{A}$ ), and $\mathscr{A}^{\prime \prime}$ the commutant of $\mathscr{A}^{\prime}$, one easily shows the additional relation $\mathscr{A}^{\prime \prime} \supset C_{2}(\mathscr{A})$. With this notation the question under consideration concerns the validity of the equation $C_{2}(\mathscr{A})=\mathscr{A}$. This can be conveniently split into $C_{2}(\mathscr{A})=C_{\infty}(\mathscr{A})$ and $C_{\infty}(\mathscr{A})=\mathscr{A}$. The first of these has received some study. For example, Jacobson has shown that if $C_{2}(\mathscr{A})$ is the algebra of all linear transformations, then so is $C_{\infty}(\mathscr{A})$ [6, p. 60]. More generally, if $\mathscr{A}$ is completely reducible (every invariant subspace has an invariant complement), then $\mathscr{A}^{\prime \prime}=C_{\infty}(\mathscr{A})[1, \S 4$, Theorem 1], so that $C_{2}(\mathscr{A})=C_{\infty}(\mathscr{A})$ by the observation above.

2. We now consider several situations in which it follows from $\mathscr{L}(\mathscr{A}) \subset$ $\mathscr{L}(T)$ that $T$ is a polynomial in $A$. For finite-dimensional spaces, a complete analysis of transformations $A$ with this property is carried out in [3].

For example, let $A$ be a bounded linear transformation on a Banach space, and suppose that $A$ is similar to a transformation of the form 
$B \oplus B$. If $\mathscr{L}(A) \subset \mathscr{L}(T)$, it is easy to see that $T$ must be simultaneously similar to a transformation of the form $S \oplus S$, so that $\mathscr{L}\left(B^{(2)}\right) \subset \mathscr{L}\left(S^{(2)}\right)$, and the theorem implies that $T$ is a polynomial in $A$.

In another direction, it is known [2, Theorem 10] that on a finitedimensional space, if $\mathscr{L}(A) \subset \mathscr{L}(T)$ and $T$ commutes with $A$, then $T$ is a polynomial in $A$.

THEOREM 2. Let $A$ and $T$ be linear transformations on a Banach space, with $A$ bounded. If $\mathscr{L}(A) \subset \mathscr{L}(T)$ and $T$ commutes with $A$, then $T$ is a polynomial in $A$.

Proof. Suppose first that $A$ is algebraic. For any vectors $x$ and $y$, let $M_{x, y}$ be the subspace consisting of all $r(A) x+s(A) y$, where $r$ and $s$ are polynomials. Then $M_{x, y}$ is invariant for both $A$ and $T$, and their restrictions continue to satisfy the hypotheses. Since $M_{x, y}$ is finitedimensional there is by [2, Theorem 10] a polynomial $p$ such that $T=p(A)$ on $M_{x, y}$. In particular, $T=p(A)$ on the span of $x$ and $y$. Because $x$ and $y$ are arbitrary, Theorem 1 implies that $T$ is a polynomial in $A$.

If $A$ is not algebraic, then it is not locally algebraic, and hence there is a vector $x_{0}$ such that $p(A) x_{0}=0$ only for $p=0$. Fix a polynomial $p_{0}$ with $T x_{0}=p_{0}(A) x_{0}$. We show $T=p_{0}(A)$. For any vector $y$ there are polynomials $r$ and $s$ such that $T y=r(A) y$ and $T\left(x_{0}+y\right)=s(A)\left(x_{0}+y\right)$. Then

$$
\left(p_{0}(A)-s(A)\right) x_{0}=(s(A)-r(A)) y ;
$$

call this vector $z$. If $z=0$ then $p_{0}=s$ and $s(A) y=r(A) y$, so that $T y=p_{0}(A) y$. If $z \neq 0$, note first that $p(A) z=0$ only for $p=0$; since $T$ commutes with $A$ we get

$$
T z=p_{0}(A) z=r(A) z,
$$

so that $p_{0}=r$ and $T y=p_{0}(A) y$ as before. Because $y$ is arbitrary, $T=p_{0}(A)$ as asserted.

We conclude with a result suggested by $\mathrm{P}$. Rosenthal. In his paper [5] with H. Radjavi, it is shown that in certain circumstances, if $\mathscr{A}$ is an algebra of bounded linear transformations on a Hilbert space such that $\mathscr{A}^{*}$ leaves invariant every closed invariant subspace of $\mathscr{A}$, then $\mathscr{A}$ is selfadjoint.

THEOREM 3. If $A$ is a bounded linear transformation on a Hilbert space such that $\mathscr{L}(A) \subset \mathscr{L}\left(A^{*}\right)$, then $A^{*}$ is a polynomial in $A$. In particular, $A$ is normal.

Proof. By Theorem 2 it is enough to show that $A^{*} A x=A A^{*} x$ for every vector $x$. Let $M_{x}=\{p(A) x \mid p$ polynomial $\}$. 
Assume first that $M_{x}$ is finite-dimensional. The restrictions of $A$ and $A^{*}$ to $M_{x}$ inherit the hypothesis; on choosing an orthonormal basis for $M_{x}$ so that the matrix of $A \mid M_{x}$ is triangular, this is seen to imply that the matrix is actually diagonal, and hence that $A^{*} A x=A A^{*} x$.

If $M_{x}$ is infinite-dimensional, then $p(A) x=0$ implies $p=0$. By hypothesis there are polynomials $r, s$, and $t$ such that $A^{*} x=r(A) x, A^{*} A x=s(A) A x$, and $A^{*}(A x-\lambda x)=t(A)(A x-\lambda x)$. Hence

$$
\begin{aligned}
(s(A) A-\lambda r(A)) x & =t(A)(A-\lambda) x, \\
z s(z)-\lambda r(z) & =t(z)(z-\lambda),
\end{aligned}
$$

and $s(\lambda)=r(\lambda)$ for all $\lambda \neq 0$. Consequently $s=r$ and $A^{*} A x=s(A) A x=$ $r(A) A x=A A^{*} x$ as required.

\section{REFERENCES}

1. N. Bourbaki, Eléments de mathématique. XXIII. Part 1. Les structures fondamentales de l'analyse. Livre II: Algèbre. Chap. 8: Modules et anneaux semi-simples, Actualités Sci. Indust., no. 1261, Hermann, Paris, 1958. MR 20 \#4576.

2. L. Brickman and P. A. Fillmore, The invariant subspace lattice of a linear transformation, Canad. J. Math. 19 (1967), 810-822. MR 35 \#4242.

3. J. Deddens and P. A. Fillmore, Reflexive linear transformations, J. Linear Algebra and Appl. (to appear).

4. I. Kaplansky, Infinite abelian groups, rev. ed., University of Michigan Press, Ann Arbor, Mich., 1969. MR 38 \#2208.

5. H. Radjavi and P. Rosenthal, $A$ sufficient condition that an operator algebra be self-adjoint, Canad. J. Math. 23 (1971), 588-597.

6. C. E. Rickart, General theory of Banach algebras, University Series in Higher Math., Van Nostrand, Princeton, N.J., 1960. MR 22 \#5903.

Department of Mathematics, Dalhousie University, Halifax, Nova Scotia, CANADA 